\title{
Association of hypothyroidism with unruptured cerebral aneurysms: a case-control study
}

\author{
Kunakorn Atchaneeyasakul, MD, ${ }^{1}$ Anita Tipirneni, MD, ${ }^{1}$ Tony Zhang, BS, ${ }^{1}$ Priyank Khandelwal, MD, \\ Sudheer Ambekar, MD, ${ }^{2}$ Brian Snelling, MD, ${ }^{2}$ Sushrut Dharmadhikari, MD, ${ }^{1}$ Chuanhui Dong, PhD, ${ }^{1}$ \\ Luis Guada, MD, ${ }^{1}$ Kevin Ramdas, MD, ${ }^{1}$ Seemant Chaturvedi, MD, ${ }^{1}$ Tatjana Rundek, MD, PhD, ${ }^{1}$ and \\ Dileep R. Yavagal, MD ${ }^{1,2}$
}

Departments of ${ }^{1}$ Neurology and ${ }^{2}$ Neurosurgery, University of Miami Miller School of Medicine, Miami, Florida

OBJECTIVE Thyroid disorder has been known to affect vascular function and has been associated with aortic aneurysm formation in some cases; however, the connection has not been well studied. The authors hypothesized that hypothyroidism is associated with the formation of cerebral aneurysms.

METHODS The authors performed a retrospective case-control study of consecutive patients who had undergone cerebral angiography at an academic, tertiary care medical center in the period from April 2004 through April 2014. Patients with unruptured aneurysms were identified from among those who had undergone 3-vessel catheter angiography. Agematched controls without cerebral aneurysms on angiography were also identified from the same database. Patients with previous subarachnoid hemorrhage or intracranial hemorrhage were excluded. History of hypothyroidism and other risk factors were recorded.

RESULTS Two hundred forty-three patients with unruptured cerebral aneurysms were identified and age matched with 243 controls. Mean aneurysm size was $9.6 \pm 0.8 \mathrm{~mm}$. Hypothyroidism was present in 40 patients (16.5\%) and 9 matched controls (3.7\%; adjusted OR 3.2, 95\% Cl 1.3-7.8, $p=0.01)$. Subgroup analysis showed that men with hypothyroidism had higher odds of an unruptured cerebral aneurysm diagnosis than the women with hypothyroidism, with an adjusted OR of $12.7(95 \% \mathrm{Cl} 1.3-121.9)$ versus an OR of $2.5(95 \% \mathrm{Cl} 1.0-6.4)$ on multivariate analysis.

CONCLUSIONS Hypothyroidism appears to be independently associated with unruptured cerebral aneurysms, with a higher effect seen in men. Given the known pathophysiological associations between hypothyroidism and vascular dysfunction, this finding warrants further exploration.

https://thejns.org/doi/abs/10.3171/2016.10.JNS161953

KEY WORDS cerebral aneurysm; hypothyroidism; cerebral angiography; subarachnoid hemorrhage; vascular disorders

$\mathrm{C}$ EREBRAL aneurysms are common vessel wall defects of cerebral arteries with a prevalence of approximately $3 \%$ in the adult population. ${ }^{11}$ Most aneurysms are asymptomatic; however, the risk of rupture per year is reported to be $0.05 \%-1.9 \%{ }^{2,8}$ Several risk factors have been associated with aneurysm formation, including cigarette smoking, hypertension, estrogen deficiency, and family history of cerebral aneurysm. ${ }^{5,9,12}$ However, novel risk factors, if found, could impact prevention and perhaps the management of aneurysms.

Hypothyroidism is a common medical problem that has been associated with endothelial dysfunction, with increased extracellular matrix mucopolysaccharide deposition in vessel walls, and with abdominal aneurysm formation. ${ }^{3,4,6}$ As cerebral aneurysms are associated with the loss of arterial wall integrity from endothelium dysfunction and disorganized extracellular matrix, hypothyroidism may be associated with their formation as well. In our routine clinical practice, we observed that several patients with unruptured aneurysms had a history of hypothyroidism. Hence, we undertook a systematic retrospective study at our university medical center to test our hypothesis that hypothyroidism is associated with the formation of cerebral aneurysms. 
TABLE 1. Univariate and multivariate analysis of the odds of an unruptured cerebral aneurysm diagnosis

\begin{tabular}{|c|c|c|c|c|c|}
\hline \multirow[b]{2}{*}{ Variable } & \multirow[b]{2}{*}{ Cases } & \multirow[b]{2}{*}{ Controls } & \multirow{2}{*}{$\begin{array}{l}\text { Univariate Analysis } \\
\text { OR }(95 \% \mathrm{Cl})\end{array}$} & \multicolumn{2}{|c|}{ Multivariate Analysis } \\
\hline & & & & OR $(95 \% \mathrm{Cl})$ & $\mathrm{p}$ Value \\
\hline No. & 243 & 243 & & & \\
\hline Mean age in yrs & $60.6 \pm 1.3$ & $62.2 \pm 1.3$ & - & & \\
\hline Hypothyroidism & $16.5 \%$ & $3.7 \%$ & $5.1(2.4-10.8)^{*}$ & $3.2(1.3-7.8)$ & $0.01^{*}$ \\
\hline Female sex & $71.2 \%$ & $35 \%$ & $4.6(3.1-6.7)^{*}$ & $2.9(1.9-4.6)$ & $<0.001^{*}$ \\
\hline Hypertension & $67.4 \%$ & $85.8 \%$ & $0.3(0.2-0.6)^{*}$ & $0.7(0.4-1.2)$ & 0.2 \\
\hline Smoking history & $30.8 \%$ & $28.4 \%$ & $1.1(0.7-1.7)$ & & \\
\hline Diabetes mellitus & $19.3 \%$ & $39.5 \%$ & $0.4(0.2-0.6)^{*}$ & $0.6(0.4-1)$ & 0.05 \\
\hline Hyperlipidemia & $36.5 \%$ & $66.5 \%$ & $0.3(0.2-0.4)^{*}$ & $0.5(0.3-0.9)$ & $0.009^{*}$ \\
\hline Alcohol abuse & $8.8 \%$ & $7.8 \%$ & $1.1(0.6-2.3)$ & & \\
\hline Illicit drug abuse & $4.4 \%$ & $5.3 \%$ & $0.8(0.3-2.0)$ & & \\
\hline Family history of cerebral aneurysm & $10 \%$ & $1 \%$ & $11.4(2.7-49)^{*}$ & $8.7(1.9-40)$ & $0.005^{*}$ \\
\hline
\end{tabular}

\section{Methods}

We performed a retrospective case-control study of consecutive patients who had undergone cerebral angiography at our academic, tertiary care medical center in the period from April 2004 through April 2014. Our study received approval from our institutional review board. Inclusion criteria included consecutive patients who underwent at least 3-vessel cerebral angiography for any medical reason. Exclusion criteria included a history of ruptured aneurysm, subarachnoid hemorrhage, intracranial hemorrhage, or cerebral vessel occlusion, as these can obscure the visualization of aneurysms.

Patient demographic information, including sex, age at diagnosis, medical comorbidities (hypertension, diabetes mellitus, and hyperlipidemia), family history of cerebral aneurysm, history of smoking, history of alcohol abuse, and history of illicit drug abuse, were obtained from the electronic medical records. In addition, history of hypothyroidism and treatment were assessed.

Cases eligible for study were those with angiographic evidence of unruptured cerebral aneurysm. Diagnosis and location of cerebral aneurysm were determined by cerebral vessel digital subtraction angiography. The control group was selected from an electronic database of patients who, during the defined time period, had undergone for any medical reason at least 3-vessel diagnostic cerebral angiography with no evidence of aneurysm. Each aneurysm case was age matched ( \pm 3 years) with a randomly selected control within that age range, per a ratio of 1:1. The exclusion criterion for controls was a prior diagnosis of intracranial hemorrhage. In addition, we stratified cases and controls based on sex, as women are known to have a higher incidence of aneurysms and hypothyroidism.

Statistical analysis was performed using the Statistical Package for the Social Sciences (SPSS, version 22, IBM Corp.). The odds ratio of an angiographic diagnosis of unruptured cerebral aneurysm associated with a medical history of hypothyroidism and the $95 \%$ confidence interval were calculated. Multiple logistic regression was applied to test for potential interactions between hypothyroidism and other listed variables with statistically significant odds ratios. To confirm the association of hypothyroidism with unruptured cerebral aneurysm, a separate propensity score matched case-control matching was performed, matching for demographic and risk factors associated with cerebral aneurysm, including age, sex, hypertension, diabetes mellitus, hyperlipidemia, smoking history, and family history of aneurysm. ${ }^{1,12}$ Student's t-test and chi-square test were used as appropriate for between-group comparisons. Statistical significance was designated as a $\mathrm{p}$ value $<0.05$.

\section{Results}

For the defined study period, we identified 4889 angiography procedures performed at our institution. Among these procedures were 243 consecutive cases with angiographically confirmed aneurysms eligible for study and 243 age-matched controls. In the cerebral aneurysm group, $66.7 \%$ had anterior circulation aneurysms, $23.5 \%$ had posterior circulation aneurysms, and $9.9 \%$ had anterior and posterior circulation aneurysms; $24.8 \%$ had multiple aneurysms. Mean aneurysm size was $9.6 \pm 0.8 \mathrm{~mm}$. Diagnoses in the control group were intracranial and/or extracranial atherosclerosis $(45.3 \%)$, arteriovenous fistula (13.2\%), vasculitis (3.3\%), arteriovenous malformation (1.6\%), other (12.8\%), and nondiagnostic (23.9\%).

The results of univariate analysis of demographics and medical history are shown in Table 1. Hypothyroidism was present in 40 patients with unruptured cerebral aneurysms $(16.5 \%)$ and 9 matched controls (4.3\%). Results of multivariate analysis, including the variables hypothyroidism, female sex, hypertension, diabetes mellitus, hyperlipidemia, and family history of cerebral aneurysm, are also shown in Table 1. Hypothyroidism was a significant independent risk factor for an unruptured cerebral aneurysm diagnosis (OR 3.2, 95\% CI 1.3-7.8, p = 0.01). Levothyroxine therapy was ongoing in $89.2 \%$ of patients with hypothyroidism in the case group compared with $100 \%$ in the control group $(p=0.3)$. Female sex was also independently associated with increased odds of an unruptured cerebral aneurysm diagnosis (OR 2.9, 95\% CI 1.9-4.6, p < 0.001), 
TABLE 2. Stratified analysis by sex to evaluate hypothyroidism and odds of unruptured cerebral aneurysm diagnosis

\begin{tabular}{crrcc}
\hline Hypothyroidism & $\begin{array}{c}\text { Cases } \\
(\mathrm{n}=243)\end{array}$ & $\begin{array}{c}\text { Controls } \\
(\mathrm{n}=243)\end{array}$ & $\begin{array}{c}\text { Crude OR } \\
(95 \% \mathrm{Cl})\end{array}$ & $\begin{array}{c}\text { Adjusted OR } \\
(95 \% \mathrm{Cl})\end{array}$ \\
\hline Male & & & & \\
\hline No & 64 & 156 & Reference & Reference \\
\hline Yes & 6 & 2 & $7.3(1.4-37.2)$ & $12.7(1.3-121.9)$ \\
\hline Female & & & & \\
\hline No & 139 & 78 & Reference & Reference \\
\hline Yes & 34 & 7 & $2.7(1.2-6.4)$ & $2.5(1.0-6.4)$ \\
\hline
\end{tabular}

as was a family history of cerebral aneurysm (OR 8.7, 95\% CI $1.9-40, p=0.005$ ). Hyperlipidemia was independently associated with decreased odds of an unruptured diagnosis (OR $0.5,95 \%$ CI $0.3-0.9, \mathrm{p}=0.009)$. There was no multiplicative effect between hypothyroidism and family history of cerebral aneurysm on the odds of an aneurysm diagnosis. Multiple studies have found smoking history to be associated with cerebral aneurysms; thus, we performed another multivariate analysis including smoking history as a potential confounder. After including smoking history, hypothyroidism was still independently associated with increased odds of an aneurysm diagnosis (OR $4.3,95 \%$ CI $1.8-10.4, \mathrm{p}=0.001$ ).

A separate propensity score matched case-control matching for demographic and known risk factors associated with cerebral aneurysm, including age, sex, hypertension, diabetes mellitus, hyperlipidemia, smoking history, and family history of aneurysm, was performed. Seventythree cases were matched with 73 controls. There was no significant difference in age, sex, hypertension, diabetes mellitus, hyperlipidemia, smoking history, and family history of aneurysm between cases and controls. Hypothyroidism was independently associated with increased odds of an aneurysm diagnosis (OR 3.2, 95\% CI 1.1-9.5).

Results of sex-stratified analyses of hypothyroidism and the odds of an unruptured cerebral aneurysm diagnosis are shown in Table 2. Odds ratios were adjusted by applying multivariate analysis to hypertension, diabetes, hyperlipidemia, and family history of cerebral aneurysm. Men with hypothyroidism were found to have higher odds of an unruptured cerebral aneurysm diagnosis than women with hypothyroidism, with an adjusted OR of 12.7 (95\% CI 1.3-121.9) versus an OR of 2.5 (95\% CI 1.0-6.4) on multivariate analysis.

Table 3 shows the comparison of unruptured cerebral aneurysms in patients with versus without a history of hypothyroidism. Unruptured cerebral aneurysms in hypothyroidism patients did not have significantly different dimensions (neck and height), numbers of lobes, or numbers of aneurysms compared with patients with no history of hypothyroidism.

\section{Discussion}

Hypothyroidism has been associated with vascular endothelial dysfunction due to impaired metabolism, causing abnormal mucopolysaccharide deposition in vessel
TABLE 3. Comparison of unruptured cerebral aneurysms in patients with versus without a history of hypothyroidism

\begin{tabular}{lccc}
\hline \multicolumn{1}{c}{ Variable } & $\begin{array}{c}\text { History of } \\
\text { Hypothyroidism } \\
(\mathrm{n}=40)\end{array}$ & $\begin{array}{c}\text { No History of } \\
\text { Hypothyroidism } \\
(\mathrm{n}=203)\end{array}$ & $\begin{array}{c}\mathrm{p} \\
\text { Value }\end{array}$ \\
\hline Neck size $(\mathrm{cm})$ & $5.2 \pm 0.9$ & $4.4 \pm 0.3$ & 0.1 \\
\hline Max dimension $(\mathrm{cm})$ & $10.4 \pm 2$ & $9.5 \pm 0.9$ & 0.403 \\
\hline No. of lobes & $1 \pm 0.1$ & $1.1 \pm 0.03$ & 0.804 \\
\hline No. of aneurysms & $1.5 \pm 0.2$ & $1.3 \pm 0.1$ & 0.302 \\
\hline
\end{tabular}

walls and perhaps associated with aneurysm formation. ${ }^{3,10}$ This retrospective, single-center, case-control study suggests that a history of hypothyroidism is independently associated with unruptured cerebral aneurysms.

Other epidemiological studies of unruptured cerebral aneurysms have not addressed hypothyroidism as a possible risk factor.,12 However, hypothyroidism has been found to be associated with aortic aneurysms. ${ }^{10}$ Some novel risk factors that have been studied and found to be associated with a decreased risk of cerebral aneurysm include hyperlipidemia and diabetes mellitus, as in our casecontrol study. ${ }^{12}$ A history of smoking has been associated with the risk of cerebral aneurysm in the literature. ${ }^{12}$ In our study, there was a slightly higher percentage of smokers in the aneurysm group (30.8\%) than in the control group (28.4\%), but the difference between the 2 groups was without statistical significance; a greater number of subjects in the control group may have been needed to see the same significance published in the literature.

Further subgroup regression analysis stratified by sex showed that men with hypothyroidism have higher odds of an unruptured cerebral aneurysm diagnosis than women. Even though women are known to have higher risks of developing hypothyroidism and cerebral aneurysm, with prevalences similar to those in our patient cohort, data in our study suggest that men with hypothyroidism may be more prone to developing cerebral aneurysms.

Limitations of this study include its retrospective design. The size of our study as well as our use of data from a single institution raises the question of the generalizability of our findings; however, multivariate analysis controlling for demographics and risk factors did reveal significant associations with cerebral aneurysms. Thyroid function tests at the time of cerebral aneurysm diagnosis were not performed.

Our finding needs to be confirmed in larger prospective studies as it has several implications for clinical practice and cerebral aneurysm research. As there is evidence that thyroid replacement therapy can improve endothelial function, this treatment could be studied as a possible intervention for cerebral aneurysms.?

\section{Conclusions}

Hypothyroidism is independently associated with increased odds of an unruptured cerebral aneurysm diagnosis, with a greater effect seen in men. Given the known pathophysiological associations between hypothyroidism 
and vascular endothelial dysfunction, further study and exploration are warranted.

\section{References}

1. de la Monte SM, Moore GW, Monk MA, Hutchins GM: Risk factors for the development and rupture of intracranial berry aneurysms. Am J Med 78:957-964, 1985

2. International Study of Unruptured Intracranial Aneurysms Investigators: Unruptured intracranial aneurysms - risk of rupture and risks of surgical intervention. N Engl J Med 339:1725-1733, 1998 (Erratum in N Engl J Med 340:744, 1999)

3. Józsa L, Szederkényi J: The mucopolysaccharide content of the human aorta in hypothyroidism. Endokrinologie 50:116-122, 1966

4. La Vignera S, Condorelli R, Vicari E, Calogero AE: Endothelial dysfunction and subclinical hypothyroidism: a brief review. J Endocrinol Invest 35:96-103, 2012

5. Longstreth WT, Nelson LM, Koepsell TD, van Belle G: Subarachnoid hemorrhage and hormonal factors in women. A population-based case-control study. Ann Intern Med 121:168-173, 1994

6. Niarchos AP, Finn R: Association between hypothyroidism and abdominal aneurysm. BMJ 4:110, 1973 (Letter)

7. Papaioannou GI, Lagasse M, Mather JF, Thompson PD: Treating hypothyroidism improves endothelial function. Metabolism 53:278-279, 2004

8. Rinkel GJ, Djibuti M, Algra A, van Gijn J: Prevalence and risk of rupture of intracranial aneurysms: a systematic review. Stroke 29:251-256, 1998

9. Ronkainen A, Hernesniemi J, Puranen M, Niemitukia L, Vanninen R, Ryynänen M, et al: Familial intracranial aneurysms. Lancet 349:380-384, 1997
10. Savage C, Deanfield JE, Jung RT: Aortic aneurysm in a patient with long-standing hypothyroidism. Postgrad Med J 58:706-707, 1982

11. Vlak MH, Algra A, Brandenburg R, Rinkel GJ: Prevalence of unruptured intracranial aneurysms, with emphasis on sex, age, comorbidity, country, and time period: a systematic review and meta-analysis. Lancet Neurol 10:626-636, 2011

12. Vlak MH, Rinkel GJ, Greebe P, Algra A: Independent risk factors for intracranial aneurysms and their joint effect: a case-control study. Stroke 44:984-987, 2013

\section{Disclosures}

Dr. Yavagal is a consultant for Medtronic.

\section{Author Contributions}

Conception and design: Yavagal, Atchaneeyasakul, Rundek. Acquisition of data: Atchaneeyasakul. Analysis and interpretation of data: Atchaneeyasakul, Tipirneni, Zhang. Drafting the article: Atchaneeyasakul, Tipirneni. Critically revising the article: Yavagal, Atchaneeyasakul, Tipirneni, Khandelwal, Ambekar, Snelling, Dharmadhikari, Guada, Ramdas, Chaturvedi, Rundek. Reviewed submitted version of manuscript: Yavagal, Atchaneeyasakul. Approved the final version of the manuscript on behalf of all authors: Yavagal. Statistical analysis: Atchaneeyasakul, Dong. Administrative/technical/material support: Atchaneeyasakul. Study supervision: Yavagal, Atchaneeyasakul.

\section{Correspondence}

Dileep R. Yavagal, University of Miami Miller School of Medicine, Department of Neurology, 1120 NW 14th St., Ste. 1366, Miami, FL 33136. email: dyavagal@med.miami.edu. 